\title{
Finite Element analysis of different composite materials using multi continuum method
}

\author{
Emayavaramban $E^{1}$, Pravin Raj E.L ${ }^{2}$ \\ ${ }^{I}$ Vice president, Imperial Society of innovate Engineers \\ ${ }^{2}$ Assistance professor, Lovely Professional University, Punjab
}

Abstract:This paper mainly deals with analysis of composite materials using Multi - continuum method. The fiberssuch as carbon fiber, glass fiber and Kevlar are mainly used. Ansys and Autodesk composite analysis is used to analysis all the fiber reinforced composites. In this paper simple composites and hybrid fiber composites were analyzed and at the end came up with optimized hybrid composite that is best suited for the given load condition.

Keywords: FEM, fiber, composites, Multi continuum method, Glass, Carbon, Kevlar, analysis, micromechanics

\section{Introduction}

Accuracy of the finite element analysis is totally based on the assumption what we take. Traditionally for composite analysis the completes composite is assumed to be a isotropic material by calculating approximate strength of the composite material, which was later proven that this method is not suitable for fibers reinforced composites. By this way the total interaction between fibers and matrix material was totally eliminated. Traditional methods shows the fiber failure earlier than the experimental prediction. The multi continuum method equip tradition structural analysis with micromechanics which has shown remarkable increase in the accuracy of results. By this we can confidently optimize the design without doing any costly experiments.

\section{Multi continuum method}

The idea of the multicontinuum mechanics was first proposed by Garnich and Hansen The concept of a multicontinuum is based on the composite to constituent stress and strain decomposition first described by Hill. The originally proposed Garnich and Hansen method was later adapted for failure analysis by Mayes and Hansen. More recently, Nelson and Hansen enveloped a modified von Mises failure criterion and provided improved solutions.
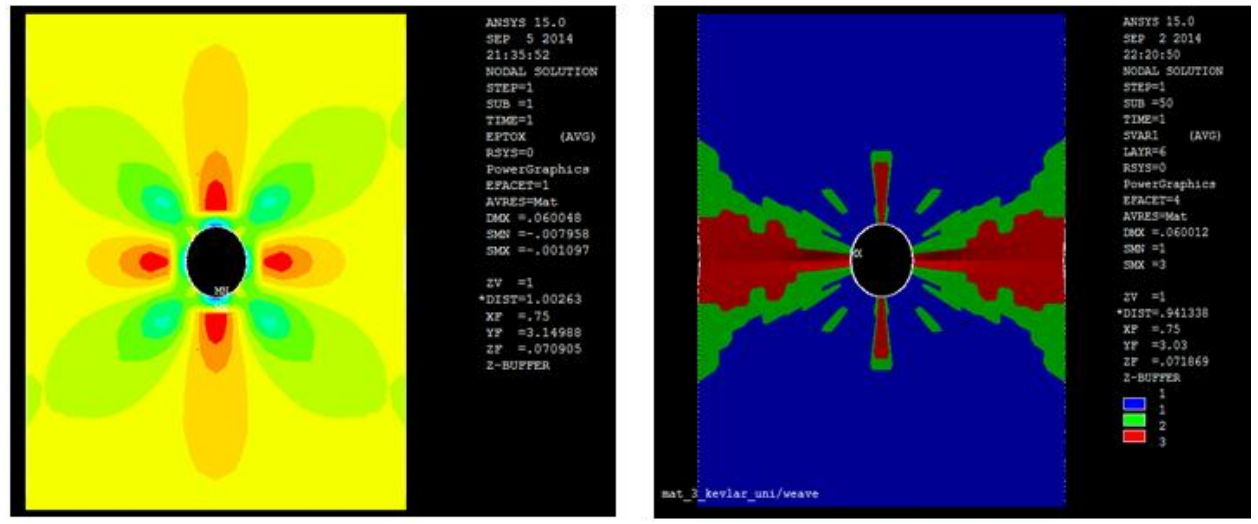

Figure 1 Analysis using traditional methodFigure 2 analysis using MCT

The foundation of the multicontinuum is to build upon traditional continuum mechanics and utilize micromechanics where proven most accurate. Traditional continuum mechanics are used to determine the stress and strain fields for a composite at a point of interest. Then using predetermined relationships between the composite and constituents, average constituent stresses and strains are decomposed from the composite results. Micromechanics analysis is used to establish the needed relationships between the composite and constituents.

\section{Material section}

Carbon fiber, glass fiber and Kevlar is selected because of their extensive use in domestic and industrial applications. Fiber reinforced composites have changed a lot in modern industrial world. Glass fiber is very cheap and its easy manufacturing method makes it best suited for economical usages but glass fiber is highly affected by humidity, wear and tear. Kevlar is used in bullet proof jackets and fuel tanks of formula one car and military vehicles. Kevlar possess very unique property, it doesn't not have much rigidity but it is very 
strong so that even it can stop bullet in its place. In other hand carbon is the most rigid than most other materials when compared with steel it is many times strong, but carbon fiber is extremely expensive so it mostly used in high performance vehicles and space equipment. Mechanical property and its matrix material is listed in table given below.

Table 1 Description of fiber used in analysis

\begin{tabular}{|c|c|c|c|c|c|c|}
\hline \multicolumn{7}{|c|}{ Properties of Fibers used $^{1}$} \\
\hline Fiber type & \multicolumn{3}{|c|}{ Unidirectional } & \multicolumn{3}{|l|}{ Weave } \\
\hline Fiber name & Glass & Carbon $^{2}$ & Kevlar & Glass & Carbon & Kevlar \\
\hline Fiber ref no. & 9036 & 9037 & 9038 & 9039 & 9040 & 9041 \\
\hline Description & S2/S0381 & $\mathrm{T} 700 / 2510$ & Kevlar-49 & $1800 / 510 \mathrm{~A}-40$ & T700/510A-40 & kavlor49 \\
\hline cured thickness & 0.233 & 0.183 & 0.28 & 0.417 & 0.625 & 0.28 \\
\hline Matrix type & epoxy & epoxy & epoxy & Vinyl Ester & Vinyl Ester & epoxy \\
\hline VolumeFraction & $72 \%$ & $49.5 \%$ & $60 \%$ & 40 & 56 & 60 \\
\hline Density $\left(\frac{\mathrm{g}}{\mathrm{cm}^{3}}\right)$ & 1.91 & 1.50 & 1.384 & 2.2 & 1.6 & 1.3 \\
\hline E11 $(\mathrm{GPa})$ & 48.6 & 125 & 75.84 & 22.2 & 56.1 & 39.625 \\
\hline E22 (GPa) & 13.6 & 8.4 & 5.5 & 20.3 & 57.1 & 39.625 \\
\hline$+\mathrm{S} 11(\mathrm{MPa})$ & 1530 & 2170 & 1378.8 & 359 & 1130 & 801 \\
\hline$+\mathrm{S} 22(\mathrm{MPa})$ & 58.5 & 48.9 & 29.64 & 315 & 1040 & 801 \\
\hline -S11 (MPa) & 1200 & 1450 & 275.76 & 436 & 450 & 161 \\
\hline -S22 (MPa) & 274 & 199 & 137.89 & 389 & 387 & 161 \\
\hline Gxy (Gpa) & 4.07 & 4.23 & 2.06 & 3.9 & 4.2 & 2.15 \\
\hline Sxy (Mpa) & 132 & 155 & 62.05 & 40.3 & 47.5 & 290 \\
\hline Sxz (Mpa) & 67.9 & 86.1 & 65.2 & 57.1 & 59.3 & 138 \\
\hline v12 & 0.263 & 0.309 & 0.34 & 0.14 & 0.07 & 5.18 \\
\hline
\end{tabular}

Materials properties were first fed into Autodesk composite analysis software. The macro given below is used to import the material properties into Ansys. In this macro command second parameter defines the material reference number which was predefined by Autodesk software.

\section{COMPAN $, 9038,7,3,1,1,0,0,0,0,0,0,0,0,0.10,0.01,0$}

\begin{tabular}{|c|c|c|c|c|c|c|c|}
\hline \multicolumn{7}{|c|}{ Table 2 layer properties } \\
\hline Layers & I and XII & II and XI & III and X & IV and IX & V and VIII & VI and VII \\
\hline Ply angle & $0^{\circ}$ & $0^{\circ}$ & $+45^{\circ}$ & $-45^{\circ}$ & $+90^{\circ}$ & $-90^{\circ}$ \\
\hline Mat_1 & 9039 & 9039 & 9036 & 9036 & 9039 & 9039 \\
\hline Mat_2 & 9040 & 9040 & 9036 & 9036 & 9040 & 9040 \\
\hline Mat_3 & 9041 & 9040 & 9037 & 9037 & 9037 & 9040 \\
\hline Mat_4 & 9041 & 9041 & 9036 & 9036 & 9040 & 9040 \\
\hline Mat_5 & 9039 & 9039 & 9038 & 9038 & 9040 & 9041 \\
\hline Mat_6 & 9040 & 9040 & 9040 & 9040 & 9040 & 9040 \\
\hline Mat_7 & 9040 & 9040 & 9039 & 9039 & 9041 & 9041 \\
\hline Mat_8 & 9037 & 9037 & 9037 & 9037 & 9037 & 9037 \\
\hline Mat_9 & 9039 & 9039 & 9040 & 9040 & 9038 & 9037 \\
\hline
\end{tabular}

Then the concern material is added to specific layer by using Ansys preprocess commands that are discussed later in this paper.Since fiber reinforced composites are anisotropic material they exhibit unique mechanical property when measured from each direction. For maintain a standard in analysis all the composites have same ply arrangement that is $\left[(+0)_{2}( \pm 45)( \pm 90)\right]_{\mathrm{s}}$. In this arrangement only the material of the fiber is changed depending on the requirement.

\section{Preprocessing and solution}

This test is based on the ASTM D3039. There was little modification made in the testing criteria, a small hole of $0.25 \mathrm{in}$ diameteris given to increase notchsensitivity. The rectangular plate of dimensions $6 \mathrm{in} \times 1.5 \mathrm{in}$ is used with thickness $0.144 \mathrm{in}$. The plate composes of 12layers each layers have thickness of $0.012 \mathrm{in}$.

\footnotetext{
${ }^{1}$ Not all the properties that are used were mentioned here. Only selected properties are listed here. For complete data refer the manufacturer data sheet. All the data are acquired for $23.9^{\circ} \mathrm{C}$.

${ }^{2}$ The name carbon most used than graphite.

${ }^{3}$ Because of the symmetry in layups, two equivalent layers were given together.
} 


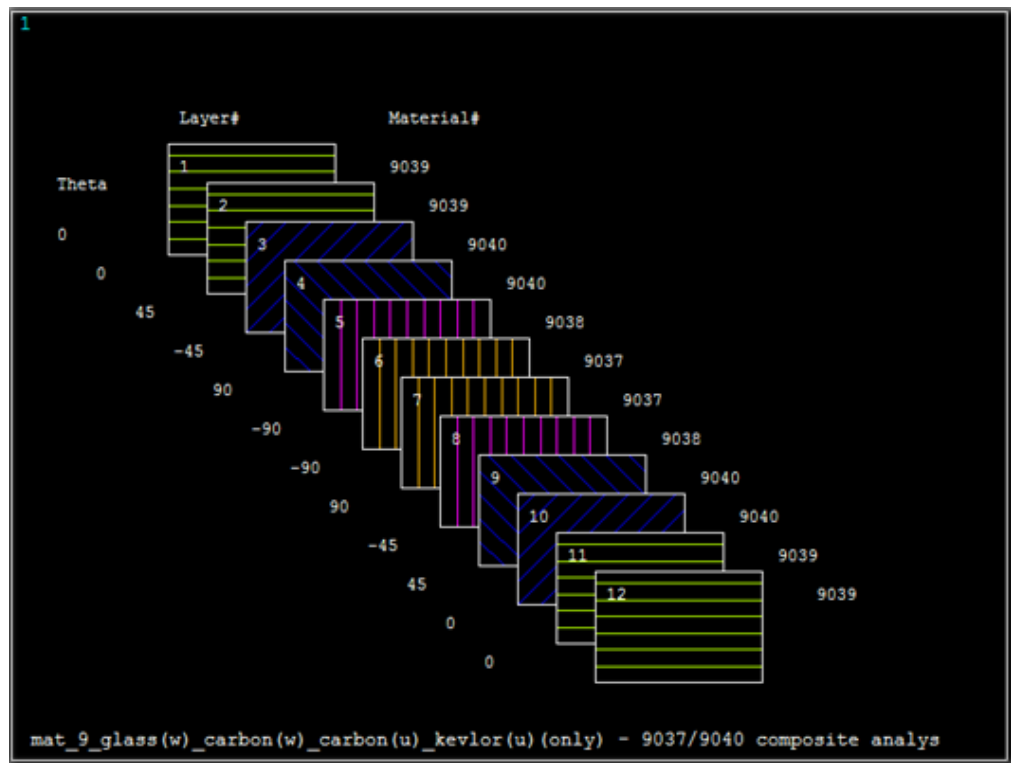

Figure 3 Section plot for mat_9

For the boundary conditions bottom part of the plate is fixed and all degree of freedom were constrained. And a constant displacement of 0.06in is provided to imitate the tensile testing environment. All the layers are defined by the given the command.

\section{SECDATA, $0.006,9040,45,3$}

The layup plot for mat_9 is shown in the figure. Here the angle for layer orientation is provided by the value theta which is given along with the given command. SOLSH190 which is 3-D 8-Node Structural Solid Shell is chosen because it combines both the properties of shell element and solid element.SOLSH190 supports layered applications such as modeling laminated shells or sandwich construction. Accuracy in modeling composite shells is governed by the firstorder shear-deformation theory (also known as Mindlin-Reissner shell theory). Keyopt were

\begin{tabular}{|c|c|c|}
\hline SVAR & Color & Description \\
\hline 1 & Blue & No failure \\
\hline 2 & Green & Matrix failure \\
\hline 3 & Red & Matrix and fiber failure \\
\hline
\end{tabular}
selected such that it supports $\mu$-p formulation. For defining a proper mesh NDIV=1 is chosen along $\mathrm{Z}$ direction in all the sides.For meshing this composite, a size of $0.075 \mathrm{in}$ is defines to get almost accurate results.

All the matrix and combined failure were

\section{Post processing.}

defined in the state variable (SVAR) by Autodesk composite analysis plugin. In the state variable three values were defined.Meaning for those values were provided in the table.Solutions were gathered using the code given below. The ESEL command selects the elements that are given in the condition. *GET command is used to count the elements that are selected by ESEL Command and save them in a variable. Then these values are saves in a user defined table LAY_SVR for processing.

The data from all the material and for all the layers were collected and processed together and the data is interpolated as the graph between layer material failures towards layer number. Percentage of failure is calculates by taking ratio of elements on failure to total number elements on the layer.

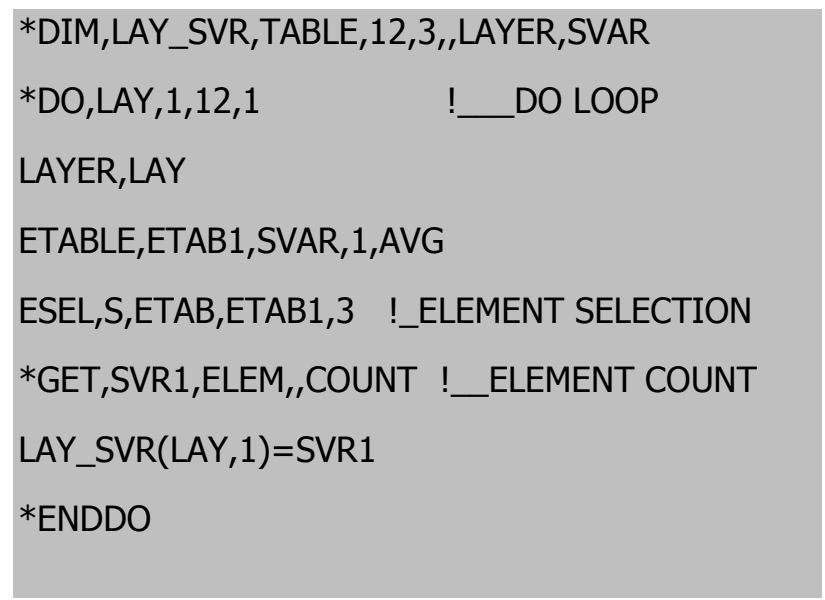


The Figure 6 and 7below shows the matrix failure percentage and combined failure percentage in each layer for mat_1,mat_3, mat_4, mat_5 and mat_7. Mat_1 and mat_3 contains totally single type of fibers, glass and Kevlar respectively. Mat_4, mat_5 and mat_7 are made up of different combinations of fibers. All the data is explained in the table given above. On checking the solution we can notice that mat_4 experiences lot of

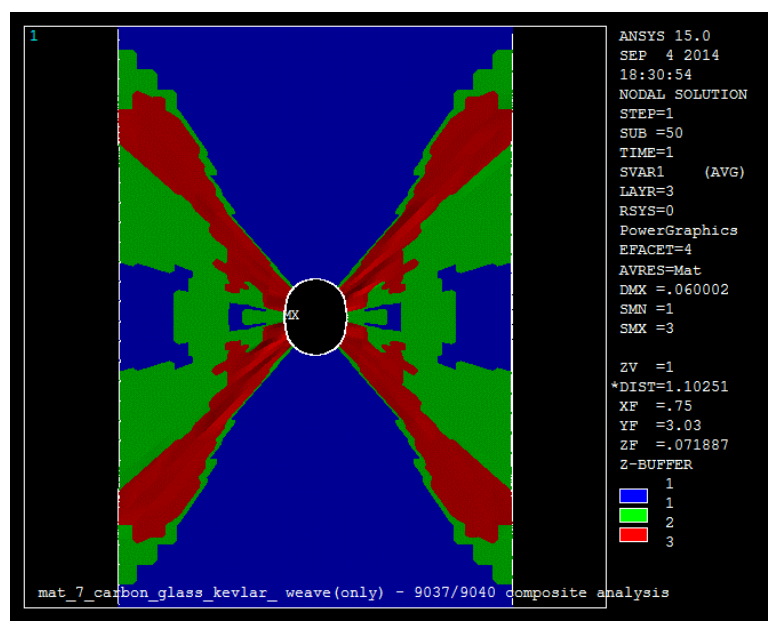

Figure 4 mat_9 layer 6

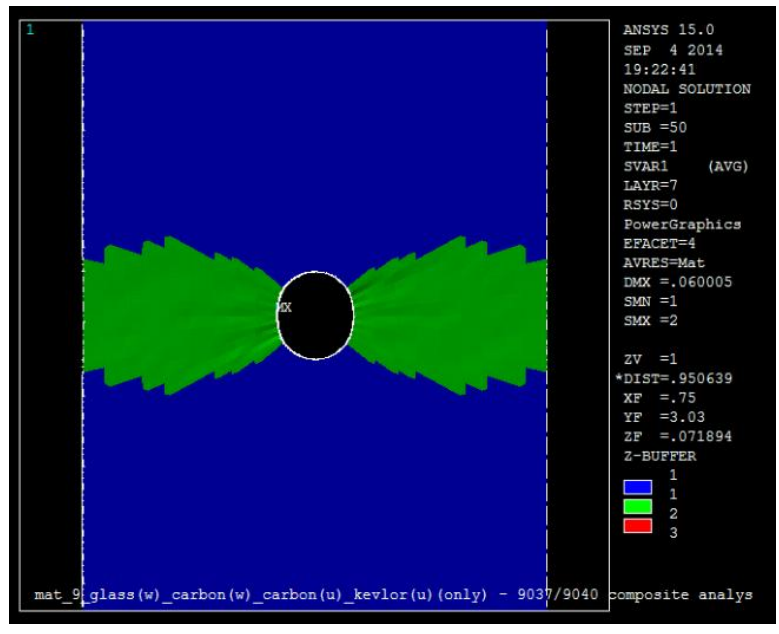

Figure 5 mat_7 layer 4

matrix failure but considerable acceptable combined failure which shows a lot of improvement is required in matrix material to make it feasible to work in this condition. In other hand mat_3 shows little uniqueproperty that its matrix failure is less than $10 \%$ and less that $2.5 \%$ for combined failure on 3 and 4 layers, which contains unidirectional carbon fiber. For mat_7 maximum matrix failure occurs on $3^{\text {rd }}$ and $4^{\text {th }}$ layer but combined failure on $1^{\text {st }}$ and $2^{\text {nd }}$ layer which shows that carbon fiber layer failure along with the matrix material. Fiber tends to take more load then transferring to the matrix layer.

The matrix and combined failure of other composite material are shown in Figure 8 and Figure 9. These composite were possess unique kind of deformation then the previous ones. Mat_2 has very low failure percentages, it has a constant failure fiber failure of around $5.2 \%$ but the component is made up of carbon fiber which is very costly comparing to other materials. Mat_6 doesn't give much impressive results because the 45 degree layer cannot takesmuch load which are made up of carbon. Mat_8 and mat_9 gives satisfactory results where mat_9 is optimized layer arrangement makes it economical as well as strong. Mat_9 even gives $0 \%$ fiber failure in some layers that is what were are expecting.

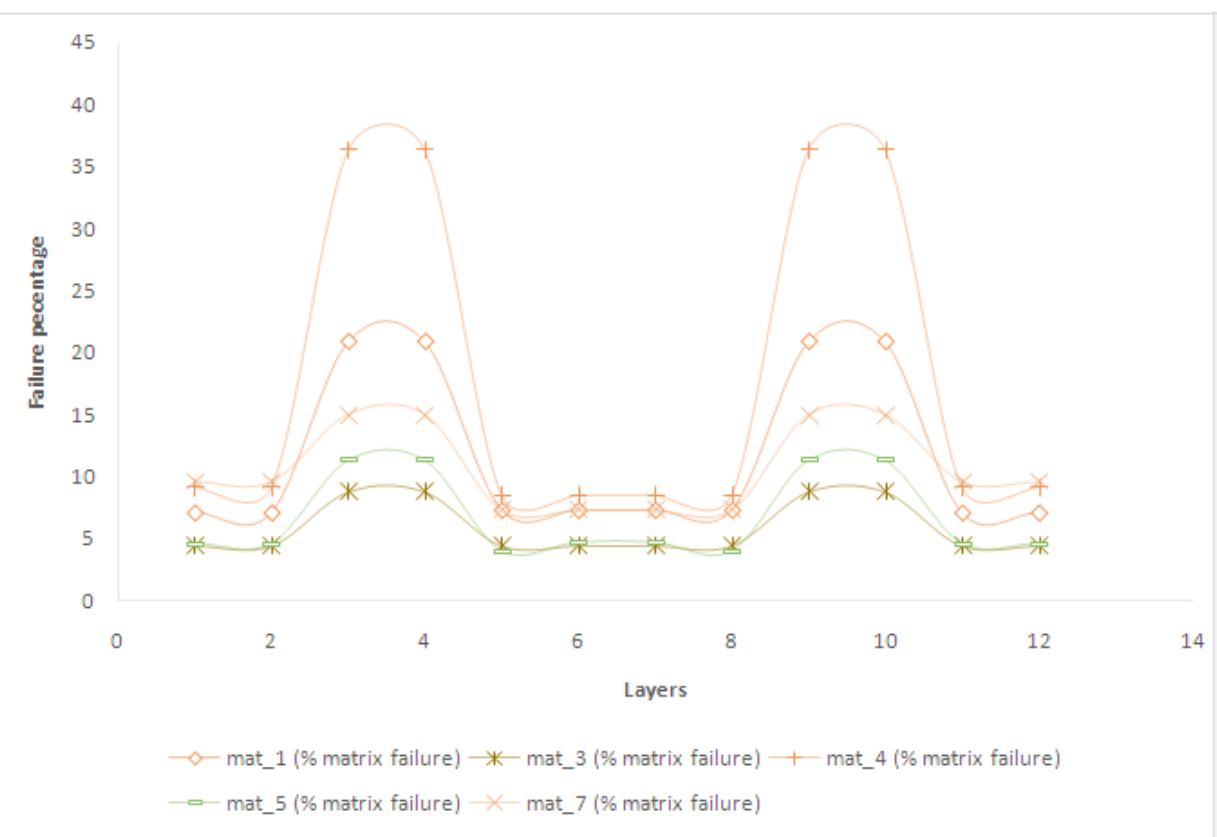

Figure 6 Graph showing matrix failure for Mat_1, Mat_3, Mat_4, Mat_5 and Mat_7 


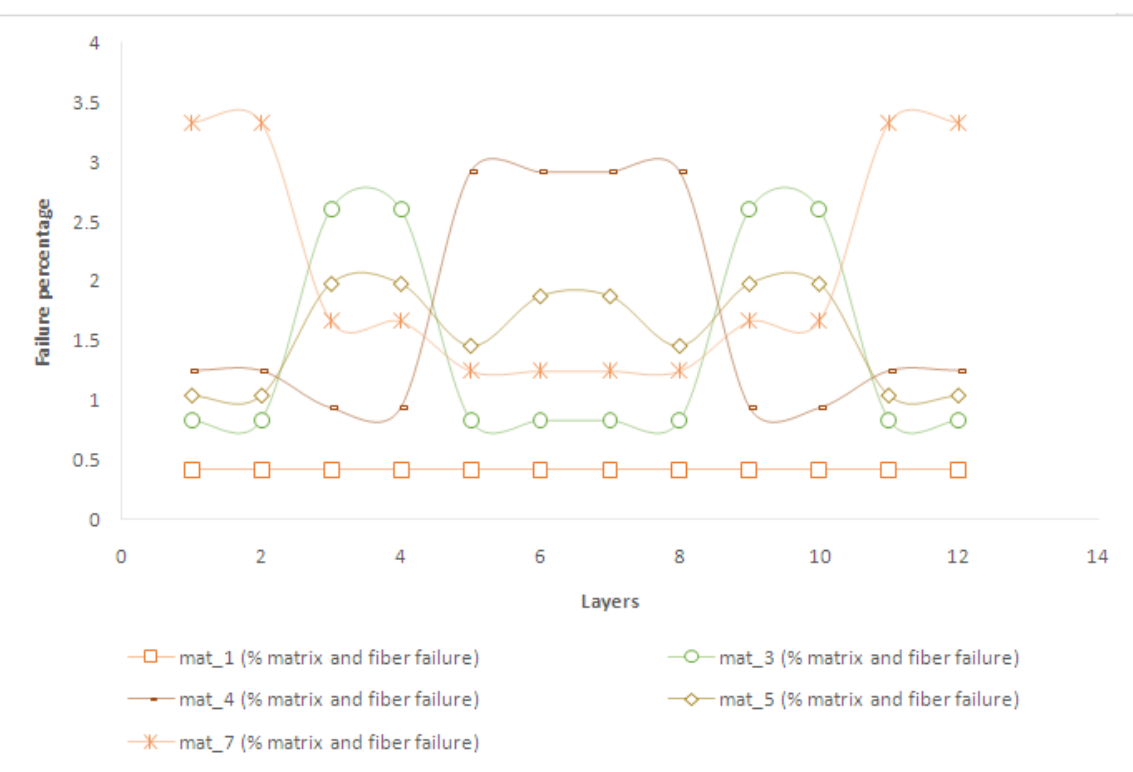

Figure 7Graph showing matrix and fiber failure for Mat_1, Mat_3, Mat_4, Mat_5 and Mat_7

\section{Chart Title}

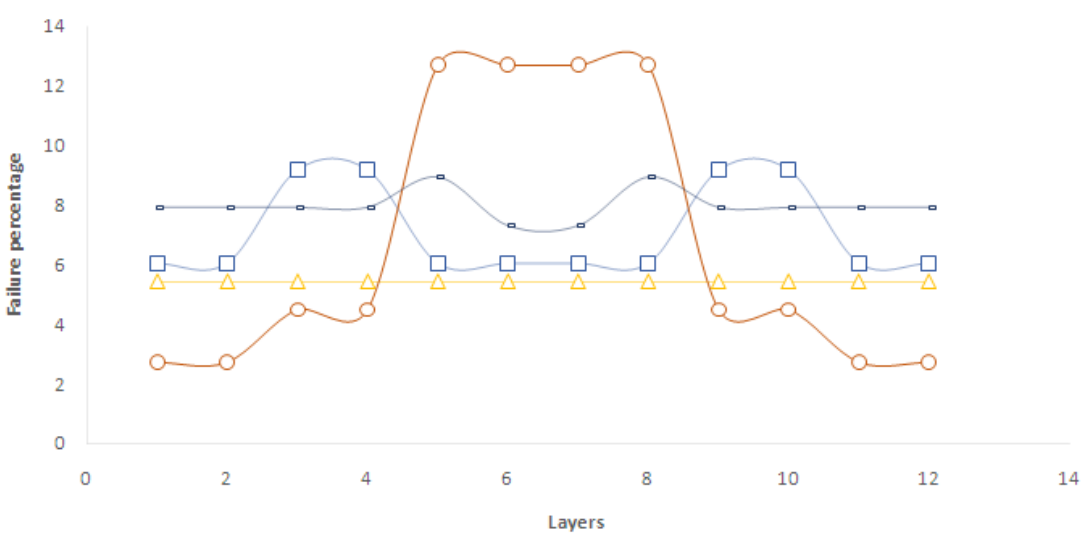

$\triangle$ mat_2 (\% matrix failure) $-\square-$ mat_6 (\% matrix failure)

$\longrightarrow$ - mat_8 (\% matrix failure) - mat_9 (\% matrix failure)

Figure 8Graph showing matrix failure for Mat_2, Mat_6, Mat_8 and Mat_9

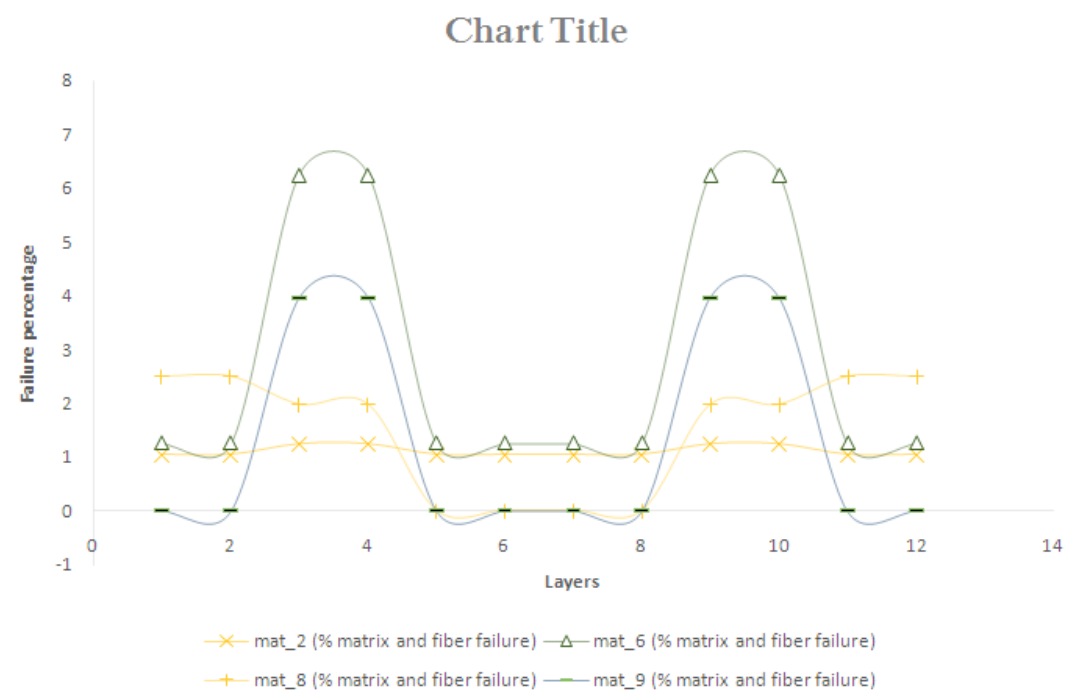

Figure 9Graph showing matrix and fiber failure for Mat_2, Mat_6, Mat_8 and Mat_9 


\section{Conclusion:}

The fiber reinforced composite material were used in the industrial field, design engineers are doing so much hard work to accurately predict the failure of composite. Since they have nonlinear properties, simulation for these composites are very hard. There are many method to solve these kind of problems but most of them fails to get the accurate result. The multi continuum method is practically best suited for composite analysis which gives complete insight about the fiber failure in all the layers of the material. This method makes the simulation of composite easy and economical which ultimately gets the best products in the market for the improvement of the human race.

\section{References}

[1]. Standard Test Method for Tensile Properties of Polymer Matrix Composite Materials, vol. 15.03, West Conshohocken, PA, 2003.

[2]. E. E. Nelson, Jason A. Gies and Ray S. Fertig III, "The Virtues of Multicontinuum Mechanics for Composites," 50th AIAA/ASME/ASCE/AHS/ASC Structures, Structural Dynamics, and Materials Conference.

[3]. M. Garnich and A.C. Hansen, "A Multicontinuum Approach to Structural Analysis of Linear Viscoelastic," J ApplMech, vol. 64, 1997.

[4]. R. Hill, "Elastic properties of reinforced solids: Some theoretical principles," Journal of the Mechanics and Physics of solids, vol. 11 , pp. 357-372, 1963

[5]. Y. Nakasone, S. Yoshimoto and T. A. Stolarski, Engineering Analysis With ANSYS Software, Elsevier Butterworth-Heinemann, 2006.

[6]. [Online]. Available: http://help.autodesk.com/view/ACMPAN/2015/. [Accessed 2092014 ].

[7]. "Fireholetech," [Online]. Available: http://composites.ides.com/.

[8]. Department of defense, Composite Materials Handbook, vol. 2, 2002.

[9]. A. C. Hansen, "Failure analysis of multi layered, multi-functional composite structures using a finite element multicontinuum theory," 2001.

[10]. K. K.Chawla, Composite materials: science and engineering, Third ed., Springer.

[11]. P.K.Mallick, Fiber Reinforced Composites, Second ed. 